Pacific Journal of Mathematics

MULTIPLIERS AND OPERATOR ALGEBRAS ON BOUNDED 


\title{
MULTIPLIERS AND OPERATOR ALGEBRAS ON BOUNDED ANALYTIC FUNCTIONS
}

\author{
MARTIN BARTELT
}

Let $B$ denote the vector space of bounded analytic functions on the open unit disc. The first part of this paper involves the use of three topologies on $B$ which give rise to various continuity classes of operators from $B$ to $B$, and the study of the relationships among these classes. The second is the examination of a special class of operators called multipliers. An operator $T$ is a multiplier if for some sequence $c_{n}$, we have $T\left(\sum a_{n} z^{n}\right)=\sum a_{n} c_{n} z^{n}$ for any function $\sum a_{n} z^{n}$ in $B$. We characterize all the multipliers from $B$ into $B$ and study their continuity properties.

2. Definitions. Let $D=\{z:|z|<1\}$ be the open unit disc in the complex plane and let $\Gamma=\{z:|z|=1\}$ be its boundary.

We shall look at the vector space $B$ in each of three different topologies, using $(B, \tau)$ to indicate the space $B$ with topology $\tau$. The topology $\kappa$ is the compact-open topology, uniform convergence on compact subsets of $D$, and $(B, \kappa)$ is a metrizable linear topological space. The topology $\sigma$ is the usual topology of uniform convergence on $D$, and $(B, \sigma)$ is a normed space with norm $\|f\|=\sup |f(z)|$ for $|z|<1$.

The strict topology $\beta$ on the vector space $B$ is the locally convex topology defined by the collection of seminorms $\|f\|_{\phi}=\|f \phi\|$ for $\phi$ a continuous function on $D$ which vanishes at infinity. The topology $\beta$ was introduced in [1] where it was shown that a sequence of functions is $\beta$ convergent if and only if it is $\sigma$ bounded and $\kappa$ convergent. The three topologies [4] are related by $\kappa \subset \beta \subset \sigma$. One advantage of the strict topology is that $(B, \beta)$, in contrast to $(B, \sigma)$, has a nice dual [4]. Also [3] the polynomials are strictly dense in $B$ whereas the uniform closure of the polynomials is just $C$, those functions in $B$ which are uniformly continuous on $D$.

Let both $\tau_{1}$ and $\tau_{2}$ be one of the three topologies $\kappa, \beta$ or $\sigma$. Then $\left[\tau_{1}: \tau_{2}\right]$ will denote the class of all continuous linear operators from $\left(B, \tau_{1}\right)$ into $\left(B, \tau_{2}\right)$. Thus $[\sigma: \sigma]$ is the algebra of all norm bounded linear operators from $(B, \sigma)$ into $(B, \sigma)$. The algebra [ $\beta: \beta]$ was essentially introduced in [2] where it was shown that $[\beta: \beta]$ is a closed subalgebra of $[\sigma: \sigma]$ in the induced norm topology.

3. Operator algebras. We have defined nine continuity classes $\left[\tau_{1}: \tau_{2}\right]$, but it will be seen that only five of them are distinct. We 
determine the inclusion relationships between the distinct classes. In particular, we show that each continuity class is an algebra and is contained in $[\sigma: \sigma]$.

Since $(B, \beta)$ is not metrizable [3], it is an important fact that a subset of $(B, \beta)$ is closed if and only if it is sequentially closed. A proof of this result for subsets which are also subspaces appears in [6]. A private communication (1966) from P. Hessler to R. C. Buck contained a proof of this result for subspaces which R. C. Buck observed also holds for arbitrary subsets. We include this unpublished proof here. Another proof is in [9]. We will use the result to conclude that a linear operator $T$ is in $[\beta: \tau]$ where $\tau$ is $\kappa, \beta$ or $\sigma$, if for any sequence $\left\{f_{n}\right\}$ converging strictly to zero, the sequence $\left\{T f_{n}\right\}$ converges $\tau$ to zero.

THEOREM 1 (Hessler). A subset of $(B, \beta)$ is closed if and only if it is sequentially closed.

Proof. Let $V$ be a sequentially closed subset of $(B, \beta)$. Let $F$ be a function which is not in $V$. We show that $F$ is not in the strict closure of $V$.

Since $V$ is sequentially closed in $(B, \beta)$ and $\beta \subset \sigma, V$ is uniformly closed. Hence there exists a $\delta>0$ such that $\|f-F\|>2 \delta$ for all $f$ in $V$. Let $\left\{K_{j}\right\}$ be a sequence of expanding compact sets whose union is all of $D$. Let $P_{n}$ be the statement that if $f$ is in $V$ and $\|f-F\| \leqq(n+1) \delta$, then there exists some integer $j \leqq n$ such that for $x$ in $K_{j}$, the maximum of $|f(x)-F(x)|>j \delta$. We show by contradiction that we can find a subsequence of $\left\{K_{j}\right\}$ such that $P_{n}$ holds for all $n$.

Statement $P_{1}$ holds vacuously for $K_{1}$. Assume that we have chosen sets $K_{1}=K(j, 1), K(j, 2), \cdots, K(j, n-1)$ and $P_{1}, P_{2}, \cdots, P_{n-1}$ all hold. Suppose that there is no compact set $K(j, n)$ for which $P_{n}$ holds. Then for any compact set $K$ after $K(j, n-1)$ in the sequence $\left\{K_{j}\right\}$, there exists a function $f_{K}$ in $V$ such that $\left\|f_{K}-F\right\| \leqq(n+1) \delta$ and for $x$ in $K_{j}$ the maximum of $\left|f_{K}(x)-F(x)\right| \leqq j \delta$ for all $j \leqq n-1$ and $\left\|f_{K}-F\right\|_{K} \leqq n \delta$. Doing this for each such compact set $K$, we obtain a sequence of functions $\left\{f_{K}\right\}$ which are uniformly bounded, since for any $K,\left\|f_{K}-F\right\| \leqq(n+1) \delta$. Then this sequence has a subsequence which converges $\kappa$ to some function $g$. Since the subsequence is uniformly bounded it also converges strictly to $g$. Since $V$ is sequentially closed, $g$ is in $V$. Denote this subsequence by $\left\{f_{k}\right\}$ and denote the corresponding compact sets by $\left\{K_{k}\right\}$. Since $\left\{f_{k}\right\}$ converges $\kappa$ to $g$ we have $\|g-F\| \leqq(n+1) \delta$. Since for each $x$ in $K_{k}$ we have $\left|f_{k}(x)-F(x)\right| \leqq k \delta$ for each $k \leqq n-1$ it follows that for each $x$ in $K_{k},|g(x)-F(x)| \leqq k \delta$ for each $k \leqq n-1$. 
Now fix a compact set $K$ occurring after $K(j, n-1)$. For any compact set $S$ containing $K$ with $S$ in $\left\{K_{k}\right\}$ we have $\left\|f_{S}-F\right\|_{K} \leqq$ $\left\|f_{S}-F\right\|_{S} \leqq n \delta$. Then as $k$ increases, the sets $K_{k}$ expand to $D$ and the functions $\left\{f_{l k}\right\}$ converge to $g$. Therefore we obtain $\|g-F\|_{K} \leqq n \delta$. We also know that for each $x$ in $K_{k},|g(x)-F(x)| \leqq k \delta$ for each $k \leqq n-1$ and that $\|g-F\| \leqq(n+1) \delta$. This contradicts $P_{n-1}$.

To complete the proof let $\phi$ be a continuous function on $D$ which vanishes at infinity such that $\phi=1 / k$ on $\partial K_{k}$, the boundary of $K_{k}$. Then the maximum of $|f(x)-F(x)|$ for $x$ in $\partial K_{k}$ is equal to the maximum of $|f(x)-F(x)|$ for $\mathrm{x}$ in $K_{k}$ which is larger than $k \delta$. Hence the maximum of $|[f(x)-F(x)] \phi(x)|$ for $x$ in $\partial K_{k}$ is larger than $\delta$. Hence $\|(f-F) \dot{\phi}\|=\|f-F\|_{\phi}>\delta$ and $F$ is not in the strict closure of $V$.

Theorem 2. The continuity classes $\left[\tau_{1}: \tau_{2}\right]$, for $\tau_{i}=\kappa, \beta$ or $\sigma$, are subsets of $[\sigma: \sigma]$.

Proof. Let $T$ be in $\left[\tau_{1}: \tau_{2}\right]$ and let the sequence $\left\{f_{n}\right\}$ converge uniformly to $f$. We apply the closed graph theorem in $(B, \sigma)$ and assume that $\lim _{n \rightarrow \infty} T f_{n}=g$, i.e. the sequence $\left\{T f_{n}\right\}$ converges uniformly to the function $g$ in $B$. Then since $\tau_{1} \subseteq \sigma,\left\{f_{n}\right\}$ converges $\tau_{1}$ to $f$. Therefore $\left\{T f_{n}\right\}$ converges $\tau_{2}$ to $T f$ and hence pointwise to $T f$. It follows that $T f=g$ and hence that $T$ is in $[\sigma: \sigma]$.

From $\kappa \subset \beta \subset \sigma$ follow some obvious inclusions among the continuity classes. We indicate which of these inclusions are proper and which continuity classes are identical.

THEOREM 3. The following identities hold for the continuity classes.

(i ) $[\sigma: \kappa]=[\sigma: \beta]=[\sigma: \sigma]$

(ii) $[\beta: \kappa]=[\beta: \beta]$

(iii) $[\kappa: \sigma]=[\kappa: \beta]$.

Proof. For the first equality we know from the last theorem that $[\sigma: \kappa] \subseteq[\sigma: \sigma]$. Since $\kappa \subset \sigma$ we have $[\sigma: \sigma] \subseteq[\sigma: \kappa]$. Therefore $[\sigma: \kappa]=[\sigma: \sigma]$. Also, since $\kappa \subset \beta$ we have $[\sigma: \kappa] \leqq[\sigma: \beta] \leqq[\sigma: \sigma]$. Since $[\sigma: \kappa]=[\sigma: \sigma]$ we are done.

For the second part let $T$ be in $[\beta: \kappa]$ and let $\left\{f_{n}\right\}$ converge strictly to zero. Then $\left\{f_{n}\right\}$ is $\kappa$ convergent and uniformly bounded. Since $T$ is in $[\beta: \kappa],\left\{T f_{n}\right\}$ is $\kappa$ convergent. Also, since $[\beta: \kappa] \subseteq[\sigma: \sigma]$, $\left\{T f_{n}\right\}$ is uniformly bounded. Therefore $\left\{T f_{n}\right\}$ converges strictly to zero.

Now let $T$ be in $[\kappa: \beta]$ and let $\left\{f_{n}\right\}$ be a sequence converging $\kappa$ to zero. Then it is known [6, pp 383] that there exists a sequence 
$\left\{c_{n}\right\}$ converging monotonically to infinity such that the sequence $\left\{c_{n} f_{n}\right\}$ converges $\kappa$ to zero. Thus $\left\{T\left(c_{n} f_{n}\right)\right\}$ converges strictly to zero. Since strictly convergent sequences are uniformly bounded, there exists a constant $M$ such that $\left\|T\left(c_{n} f_{n}\right)\right\|=c_{n}\left\|T\left(f_{n}\right)\right\| \leqq M$. Hence $\left\{T f_{n}\right\}$ converges uniformly to zero and $T$ is in $[\kappa: \sigma]$. Since $\beta \subset \sigma$, we have $[\kappa: \sigma] \subseteq[\kappa: \beta]$ and the result follows.

COROLLARY. The continuity classes $\left[\tau_{1}: \tau_{2}\right]$ are algebras.

Proof. The last theorem shows that the only possible distinct continuity classes $\left[\tau_{1}: \tau_{2}\right]$ satisfy $\tau_{1} \supseteqq \tau_{2}$. The corollary follows immediately.

THEOREM 4. Among the operator algebras $\left[\tau_{1}: \tau_{2}\right]$, the only distinct ones are $[\kappa: \sigma],[\kappa: \kappa],[\beta: \sigma],[\beta: \beta]$ and $[\sigma: \sigma]$, and all the proper inclusions between them are given by $[\kappa: \sigma] \subset[\beta: \sigma] \subset[\beta: \beta] \subset[\sigma: \sigma]$ and $[\kappa: \sigma] \subset[\kappa: \kappa] \subset[\beta: \beta]$.

Here is a simple diagram of the situation:

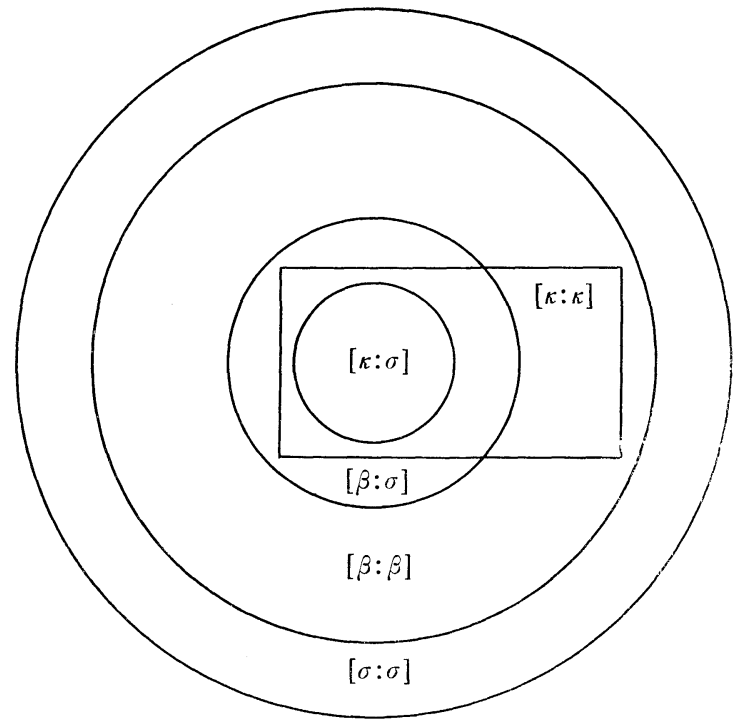

FIGURE 1

Proof. It has been shown that these are the only possible distinct classes. We also know that $[\beta: \sigma] \subseteq[\beta: \beta]$ and $[\kappa: \sigma] \subseteq[\kappa: \kappa]$. Consideration of the identity operator shows that these inclusions are proper.

To show that $[\kappa: \kappa]$ is a subset of $[\beta: \beta]$, let $T$ be a linear 
operator in $[\kappa: \kappa]$ and let $\left\{f_{n}\right\}$ be a sequence converging strictly to zero. Then $\left\{f_{n}\right\}$ converges $\kappa$ to zero and $\left\{T f_{n}\right\}$ converges $\kappa$ to zero. Also $\left\{f_{n}\right\}$ is uniformly bounded and since $[\kappa: \kappa] \subseteq[\sigma: \sigma],\left\{T f_{n}\right\}$ is uniformly bounded. Hence $\left\{T f_{n}\right\}$ converges strictly to zero.

We show that $[\kappa: \kappa]$ is a proper subalgebra of $[\beta: \beta]$ by giving an example of an operator which is in $[\beta: \sigma] \leqq[\beta: \beta]$ but not in $[\kappa: \kappa]$. This also shows that $[\beta: \sigma]$ is not contained in $[\kappa: \kappa]$. Let $T$ be the linear operator from $B$ to the constant functions defined by $\operatorname{Tf}(z)=$ $\int_{0}^{1} f(x) d x$. Then the sequence $\left\{f_{n}\right\}$, where $f_{n}(z)=n z^{n}$, converges $\kappa$ to zero but $\left\{T f_{n}\right\}$ converges to 1 . Hence $T$ is not in $[\kappa: \kappa]$. Now assume that $\left\{f_{n}\right\}$ converges strictly to zero. Then $\left\{f_{n}\right\}$ converges $\kappa$ to zero and $\left\|f_{n}\right\| \leqq M$ for some constant $M$. Let $r$ be a real number satisfying $0<r<1$. Let $\|f\|_{r}=\sup \{|f(z)|:|z| \leqq r\}$. Then

$$
\left|T f_{n}(z)\right| \leqq\left|\int_{0}^{r} f_{n}(x) d x\right|+\left|\int_{r}^{1} f_{n}(x) d x\right|
$$

Hence $\left|T f_{n}(z)\right| \leqq r\left\|f_{n}\right\|_{r}+M(1-r)$. Therefore $\left\|T f_{n}\right\|$ can be made arbitrarily small by choosing $r$ near 1 and $n$ large.

The bounded linear operator $T$ defined on $(C, \sigma)$ by $T f(z)=f(1)$ maps $C$ into the constant functions. By using the Hahn Banach theorem on the associated linear functional given by $L f=f(1)$, we can extend $T$ to a bounded linear operator on $(B, \sigma)$. But this operator $T$ is not in $[\beta: \beta]$. Although the sequence $\left\{z^{n}\right\}$ converges strictly to zero, $T\left(z^{n}\right)=1$ for all $n$.

Finally we show that $[\kappa: \sigma] \subset[\beta: \sigma]$. Since $\kappa \subset \beta$, we have $[\kappa: \sigma] \subseteq[\beta: \sigma]$. Let $T$ be the operator defined on $B$, by $T\left(\sum a_{n} z^{n}\right)=$ $\sum\left(a_{n} / n\right) z^{n}$ for any function $\sum a_{n} z^{n}$ in $B$. It follows immediately from Theorem 8 that $T$ is not in $[\kappa: \sigma]$. The corollary to Theorem 7 implies that if $T$ maps $B$ into $C$, then $T$ will be in $[\beta: \sigma]$. Since $\left(\sum\left|a_{n}\right| / n\right)^{2} \leqq \sum\left|a_{n}\right|^{2} \sum(1 / n)^{2}$, it follows that $\sum a_{n}\left(z^{n} / n\right)$ is in $C$.

4. Multipliers and diagonal operators. Assume that $T$ is a linear operator defined on $B$ for which there exists a sequence $\left\{c_{n}\right\}$ such that $T\left(z^{n}\right)=c_{n} z^{n}$, for $n=0,1 \cdots$. If $T$ is also in $[\kappa: \kappa]$, then for any function $f(z)=\sum a_{n} z^{n}$ in $B$, we have $T\left(\sum a_{n} z^{n}\right)=\sum a_{n} c_{n} z^{n}$. This holds because the partial sums of $\sum a_{n} z^{n}$ converge $\kappa$ to $f$. Operators satisfying $T\left(\sum a_{n} z^{n}\right)=\sum a_{n} c_{n} z^{n}$ for some sequence $\left\{c_{n}\right\}$ are called multipliers. The multipliers from $H^{p}$ to $H^{q}$ were first studied by Hardy and Littlewood, (for references and some recent results see [5]). Further results on multipliers can be found in [3] and [7]. Explicitly we have:

Definition. A multiplier on $B$ is a linear operator $T$ such that 
there exists a sequence $\left\{c_{n}\right\}$ with the property that $T\left(\sum a_{n} z^{n}\right)=\sum a_{n} c_{n} z^{n}$ for any function $\sum a_{n} z^{n}$ in $B$.

We distinguish another class of closely related operators which leave invariant the one dimensional subspaces generated by $z^{k}$. These operators shed light on the relationship between topology and the multipliers.

DeFinition. A linear operator $T$ defined on $B$ is called diagonal if there exists a sequence $\left\{c_{n}\right\}$ such that $T\left(z^{n}\right)=c_{n} z^{n}$, for $n=0,1, \cdots$.

Clearly any multiplier is a diagonal operator. At the beginning of this section we showed that any diagonal operator which is also in $[\kappa: \kappa]$ is a multiplier. It is not known whether there exists a diagonal operator mapping $B$ into $B$ which is not a multiplier. The action of any diagonal operator is determined by its action on the polynomials, which are $\kappa$ dense in $B$, strictly dense in $B$, but not uniformly dense in $B$. Hence it is reasonable to conjecture, although we can not prove it, that the diagonal operators lie in $[\beta$ : $\beta]$. We can show that any diagonal operator in $[\beta: \beta]$ is a multiplier.

Theorem 5. Let $T$ be a diagonal operator mapping $B$ into $B$ and assume also that $T$ is in [ $\beta$ : $\beta$ ]. Then $T$ is a multiplier from $B$ into $B$.

Proof. Since $T$ is diagonal there is a sequence $\left\{c_{n}\right\}$ such that $T\left(z^{n}\right)=c_{n} z^{n}$. We have to show that $T\left(\sum a_{n} z^{n}\right)=\sum a_{n} c_{n} z^{n}$ for any function $f(z)=\sum a_{n} z^{n}$ in $B$. For any real number $r$ with $0<r<1$, let $f_{r}(z)=f(r z)$. We first prove the theorem for the function $f_{r}$. Since $T$ is in $[\sigma: \sigma]$ and the partial sums of the power series for $f_{r}$ converge uniformly to $f_{r}$, it follows that as $N$ approaches infinity, $\sum_{n=0}^{N} a_{n} r^{n} T\left(z^{n}\right)$ converges uniformly to $T f_{r}$. Fix $x$ in $D$ and put $T\left(z^{n}\right)=u_{n}(z)$. Then

$$
\left|\sum_{n=N}^{\infty} a_{n} r^{n} u_{n}(x)\right| \leqq \sum_{n=N}^{\infty}\left|a_{n}\right| r^{n}\left|u_{n}(x)\right| \leqq\|f\|\|T\| \sum_{n=N}^{\infty} r^{n},
$$

since $\left|a_{n}\right| \leqq\|f\|$. Hence as $N$ approaches infinity, $\sum_{n=0}^{N} a_{n} r^{n} T\left(z^{n}\right)$ converges pointwise to $\sum_{n=0}^{\infty} a_{n} r^{n} T\left(z^{n}\right)$. Thus $T f_{r}(z)=\sum a_{n} r^{n} T\left(z^{n}\right)$.

Now if $f(z)=\sum a_{n} z^{n}$ is in $B$, the Cauchy integral formula shows that $f_{r}$ converges $\kappa$ to $f$ as $r$ approaches 1 through a sequence of values. Hence for any $x$ in $D$, we have

$$
T f(x)=\lim _{r \uparrow 1} T f_{r}(x)=\lim _{r \uparrow 1} \sum a_{n} r^{n} c_{n} x^{n}=\sum a_{n} c_{n} x^{n}
$$

since the function $\sum a_{n} c_{n} x^{n} w^{n}$ is analytic in $|w|<1 /|x|$. 
5. Multipliers and continuity. In this section we first characterize the multipliers from $B$ into $B$ and then determine those multipliers which lie in the various continuity classes.

The following characterization of the multipliers from $B$ into $C$, which occurs in [7], suggests how to characterize the multipliers from $B$ into $B$.

THEOREM PSW [7]. Let $T$ be a multiplier from $B$ into $C$, where $T\left(\sum a_{n} z^{n}\right)=\sum a_{n} c_{n} z^{n}$ for any function $\sum a_{n} z^{n}$ in $B$. Then the sequence $\left\{c_{n}\right\}$ is one side of the sequence of Fourier coefficients of a function in $L^{1}(\Gamma)$. Conversely, given any such sequence $\left\{c_{n}\right\}$, the operator $T$ defined on $B$ by $T\left(\sum a_{n} z^{n}\right)=\sum a_{n} c_{n} z^{n}$ is a multiplier from $B$ into $C$.

Both Theorem PSW and the next theorem on the multipliers from $B$ into $B$ can be considered as converse forms of Hadamard multiplication theorems. Let $\left\{c_{n}\right\}$ be the sequence associated with a multiplier $T$. Let $h(z)=\sum c_{n} z^{n}$. Then for any function $f(z)=\sum \alpha_{n} z^{n}$ in $B$, we have $T f(z)=\sum a_{n} c_{n} z^{n}=(h * f)(z)$, the Hadamard product of $h$ and $f$. We thus solve the problem of determining those functions $h$ such that $h * f$ is in $B$ for every $f$ in $B$.

A first step in the direction of characterizing the multipliers in various continuity algebras was taken in [3]. Let $(C, \sigma)$ be $C$ in the topology $\sigma$. Given a strictly continuous linear functional $L$ on $B$, define a linear operator $T$ on $B$ by $T\left(\sum a_{n} z^{n}\right)=\sum a_{n} L\left(z^{n}\right) z^{n}$. Then it was shown that $T$ is a continuous linear operator from $(B, \beta)$ into $(C, \sigma)$. Letting $h(z)=\sum L\left(z^{n}\right) z^{n}$, the result states that $(h * f)(z)$ is in $C$ for any function $f$ in $B$. Theorem PSW provides a converse.

Corollary. Let $h(z)=\sum c_{n} z^{n}$. Assume that $(h * f)(z)$ is in $C$ for every function $f$ in $B$. Then $c_{n}=L\left(z^{n}\right)$ for some strictly continuous linear functional $L$.

Proof. Since $h * f$ is in $C$ for any function $f$ in $B$, it follows that [7] the sequence $\left\{c_{n}\right\}$ is one side of the sequence of Fourier coefficients of a function in $L^{1}(\Gamma)$ and thus that the linear functional $L$ defined on $z^{n}$ by $L\left(z^{n}\right)=c_{n}$ can be extended to a strictly continuous functional on all of $B$.

We know that any diagonal operator in $[\kappa: \kappa]$ is a multiplier from $B$ into $B$. In fact these are all such multipliers.

THEOREM 6. Let $T$ be a multiplier from $B$ into $B$ given by $T\left(\sum a_{n} z^{n}\right)=\sum a_{n} c_{n} z^{n}$. Then there exists an $L$ in the dual of $(C, \sigma)$ 
such that $c_{n}=L\left(z^{n}\right)$, for $n=0,1, \cdots$. Conversely, given any such $L$, let $T$ be defined by $T\left(\sum a_{n} z^{n}\right)=\sum a_{n} L\left(z^{n}\right) z^{n}$. Then $T$ is in $[\kappa: \kappa]$. Furthermore $\|L\|=\|T\|$.

Proof. Let $L$ be in the dual of $(C, \sigma)$ with $L\left(z^{n}\right)=c_{n}$ and define $T$ as above. Then for fixed $x$ in $D, T f(x)=\sum a_{n} L\left(z^{n}\right) x^{n}=L\left(\sum a_{n} x^{n} z^{n}\right)$ because the partial sums of $\sum a_{n} x^{n} z^{n}$ converge uniformly for $|z|<1$. Let $U_{x}$ be the operator given by $U_{x} f(z)=f(x z)$. Then given any $r$ with $0<r<1$ and an $x$ in $D$ with $|x| \leqq r$, we have $|T f(x)| \leqq\|L\|$ $\left\|U_{x} f(z)\right\| \leqq\|L\|\|f\|_{r}$. Hence $\|T f\|_{r} \leqq\|L\|\|f\|_{r}$ and $\|T\| \leqq\|L\|$ and $T$ is in $[\kappa: \kappa]$.

Now let $T$ satisfy the conditions of the theorem and define $h(z)=\sum c_{n} z^{n}$. We show that $h(z)$ is analytic in $D$. Since the function $f(z)=\sum n^{-2} z^{n}$ is in $B$, it follows that the function $T f(z)=\sum c_{n} n^{-2} z^{n}$ is in $B$. Hence $\lim \sup \left|c_{n}\right|^{1 / n}=\lim \sup \left|n^{-2} c_{n}\right|^{1 / n} \leqq 1$.

For any $r$ with $0<r<1$, define the linear functional $L_{r}$ on $C$ by $L_{r} f=T f(r)$. Then for $f(z)=\sum a_{n} z^{n}$ in $B$, we have

$$
\left|L_{r}(f)\right|=\left|\sum a_{n} c_{n} r^{n}\right| \leqq\|f\| \sum\left|c_{n}\right| r^{n}<\infty
$$

because $\sum c_{n} z^{n}$ is analytic in $D$. Hence $L_{r}$ is in the dual of $(C, \sigma)$. Now for fixed $f$ in $C, L_{r} f$ is bounded in norm for all $0<r<1$ because $T f$ is in $B$. By the uniform boundedness principle, there is an $M$ such that $\left\|L_{r}\right\| \leqq M$ for all $0<r<1$. By the weak star compactness of the unit ball of the dual of $(C, \sigma)$ there exists an $L$ in the dual of $(C, \sigma)$ such that $L_{r} f$ converges to $L f$ for every $f$ in C. Letting $f(z)=z^{n}$ we obtain $L_{r}\left(z^{n}\right)=r^{n} c_{n}$ converging to $L\left(z^{n}\right)$ and to $c_{n}$. Hence $c_{n}=L\left(z^{n}\right)$.

Following the procedure used in the first part of the proof this $L$ now yields an operator $T$ in $[\kappa: \kappa]$. In fact this is equal to the operator which gave $L$ because it agrees with the original operator on the polynomials. From $L_{r}(f)=T f(r)$ it follows that $\left\|L_{r}\right\| \leqq\|T\|$ and hence $\|L\| \leqq\|T\|$.

Corollary. All the diagonal operators in $[\beta: \beta]$ are in $[\kappa: \kappa]$.

Any uniformly continuous linear functional on $C$ can be extended by the Hahn Banach theorem to a continuous linear functional on the space of all continuous functions on $\Gamma$. Corresponding to this functional there is a Radon measure on $\Gamma$. Hence the sequences associated with the multipliers from $B$ into $B$ correspond to the Radon measures on $\Gamma$. Since $[\beta: \sigma]$ is a subalgebra of $[\kappa: \kappa]$, the multipliers in $[\beta: \sigma]$ will correspond to some subset of the Radon measures. 
THEOREM 7. Let $T$ be a multiplier in [ $\beta: \sigma]$ given by $T\left(\sum a_{n} z^{n}\right)=$ $\sum a_{n} c_{n} z^{n}$. Then $\left\{c_{n}\right\}$ is one side of the sequence of Fourier coefficients of a function in $L^{1}(\Gamma)$. Conversely given any such sequence $\left\{c_{n}\right\}$, the operator $T$ defined by $T\left(\sum a_{n} z^{n}\right)=\sum a_{n} c_{n} z^{n}$ is a multiplier in [ $\left.\beta: \sigma\right]$.

Proof. Let $T$ be a multiplier in $[\beta: \sigma]$. For any function $f$ in $B,\left\{f_{r}\right\}$ converges $\kappa$ to $f$ and $\left\|f_{r}\right\| \leqq\|f\|$. Hence $\left\{f_{r}\right\}$ converges strictly to $f$. Therefore if $f(z)=\sum a_{n} z^{n}, T f_{r}(z)=T\left(\sum a_{n} r^{n} z^{n}\right)=$ $\sum a_{n} c_{n} r^{n} z^{n}$ is in $C$ because $\sum a_{n} c_{n} z^{n}$ is analytic in $D$. Then $\left\{T f_{r}\right\}$ converges uniformly to $T f$ which implies that $T f$ is in $C$. The result follows from Theorem PSW.

Now let $\left\{c_{n}\right\}$ be one side of the sequence of Fourier coefficients of an $L^{1}$ function. Letting $L\left(z^{n}\right)=c_{n}$, we can ([7] and [10]) extend this to a strictly continuous linear functional on $B$. As mentioned in the second paragraph after Theorem PSW, the operator $T$ defined by $T f(z)=\sum a_{n} L\left(z^{n}\right) z^{n}$ is a continuous linear operator from $(B, \beta)$ into $(C, \sigma)$.

COROLlaRy. The operator $T$ is a multiplier from $B$ into $C$ if and only if $T$ is a multiplier in $[\beta: \sigma]$.

The last class to consider is $[\kappa: \sigma]$.

THeOREM 8. Let $T$ be a multiplier in $[\kappa: \sigma]$ given by $T\left(\sum a_{n} z^{n}\right)=$ $\sum a_{n} c_{n} z^{n}$. Then $\lim \sup \left|c_{n}\right|^{1 / n}<1$. Conversely any such sequence $\left\{c_{n}\right\}$ defines a multiplier $T$ in $[\kappa: \sigma]$ given by $T\left(\sum a_{n} z^{n}\right)=\sum a_{n} c_{n} z^{n}$.

Proof. If $T$ is in $[\kappa: \sigma]$, then there exists an $r$ with $0<r<1$ and a constant $M$ such that $\|T f\| \leqq M\|f\|_{r}$ for all $f$ in $B$. Letting $f(z)=z^{n}$, we obtain $\lim \sup \left|c_{n}\right|^{1 / n} \leqq r$.

Assume now that $\lim \sup \left|c_{n}\right|^{1 / n}=c<1$. Choose an $r$ such that $c<r<1$. Then for $f(z)=\sum a_{n} z^{n}$ in $B$, Tf $(z)=\sum a_{n} c_{n} z^{n}$ which is in $C$. Then $|T f(z)| \leqq\|f\|_{r} \sum\left|c_{n}\right| r^{-n} \leqq M\|f\|$ for some constant $M$ and $T$ is in $[\kappa: \sigma]$.

We have shown that the multipliers in $[\kappa: \kappa]$ are the multipliers from $B$ into $B$ and that the multipliers in $[\beta: \sigma]$ are the multipliers from $B$ into $C$. Let $H(D)$ be the functions analytic in $D$ and let $H(\bar{D})$ be those which are analytic in some open disc containing $D$.

CoROLlary. The operator $T$ is a multiplier in $[\kappa: \sigma]$ if and only if it is a multiplier from $H(D)$ into $H(\bar{D})$. 


\section{REFERENCES}

1. R. C. Buck, Operator algebras and dual spaces, Proc. Amer. Math. Soc., 3 (1952), 681-687.

2. - Algebras of linear transformations, Technical Report \#4 under OOR contract TB2-001 (1406), (1956).

3. - Algebraic properties of classes of analytic functions, Seminars on Analytic Functions, vol. II, Princeton (1957), 175-188.

4. - Bounded continuous functions on a locally compact space, Michigan Math. J., 5 (1958), 95-104.

5. J. H. Hedlund, Multipliers of $H^{p}$ Spaces, J. Math. Mech., 18 (1969), 1067-1074.

6. G. Kothe, Topologische Lineare Raume, Springer-Verlag, Heidleberg, (1960).

7. G. Piranian, A. L. Shields and J. H. Wells, Bounded analytic functions and absolutely continuous measures, Proc. Amer. Math. Soc., 18 (1967), 818-826.

8. L. A. Rubel and A. L. Shields, The space of bounded analytic functions on a region, Ann. Ins. Fourier, (Grenoble) 16 (1966), 235-277.

9. L. A. Rubel and J. V. Ryff, The bounded weak-star topology and bounded analytic functions, J. Functional Anal., 5 (1970), 167-183.

10. A. E. Taylor, Banach spaces of functions analytic in the unit circle, II, Studia Math. 12 (1951), 25-50.

Received May 6, 1970. The research reported here was supported in part by NSF Grant GP 24182. The work is an extension of part of the author's doctoral thesis under the direction of Professor R. C. Buck at the University of Wisconsin.

Rensselaer Polytechnic Institute 


\title{
PACIFIC JOURNAL OF MATHEMATICS
}

\author{
EDITORS
}

\author{
H. SAMelson \\ Stanford University \\ Stanford, California 94305 \\ C. R. HoBBy \\ University of Washington \\ Seattle, Washington 98105
}

J. DugunduI

Department of Mathematics

University of Southern California

Los Angeles, California 90007

RICHARD ARENS

University of California

Los Angeles, California 90024

\section{ASSOCIATE EDITORS}

\author{
E. F. BeCKENBACH
}

B. H. NEUMANN

F. WoLF

K. YOSHIDA

\section{SUPPORTING INSTITUTIONS}

\author{
UNIVERSITY OF BRITISH COLUMBIA \\ CALIFORNIA INSTITUTE OF TECHNOLOGY \\ UNIVERSITY OF CALIFORNIA \\ MONTANA STATE UNIVERSITY \\ UNIVERSITY OF NEVADA \\ NEW MEXICO STATE UNIVERSITY \\ OREGON STATE UNIVERSITY \\ UNIVERSITY OF OREGON \\ OSAKA UNIVERSITY \\ UNIVERSITY OF SOUTHERN CALIFORNIA
}

\author{
STANFORD UNIVERSITY \\ UNIVERSITY OF TOKYO \\ UNIVERSITY OF UTAH \\ WASHINGTON STATE UNIVERSITY \\ UNIVERSITY OF WASHINGTON \\ AMERICAN MATHEMATICAL SOCIETY \\ CHEVRON RESEARCH CORPORATION \\ NAVAL WEAPONS CENTER
}

The Supporting Institutions listed above contribute to the cost of publication of this Journal, but they are not owners or publishers and have no responsibility for its content or policies.

Mathematical papers intended for publication in the Pacific Journal of Mathematics should be in typed form or offset-reproduced, (not dittoed), double spaced with large margins. Underline Greek letters in red, German in green, and script in blue. The first paragraph or two must be capable of being used separately as a synopsis of the entire paper. The editorial "we" must not be used in the synopsis, and items of the bibliography should not be cited there unless absolutely necessary, in which case they must be identified by author and Journal, rather than by item number. Manuscripts, in duplicate if possible, may be sent to any one of the four editors. Please classify according to the scheme of Math. Rev. Index to Vol. 39. All other communications to the editors should be addressed to the managing editor, Richard Arens, University of California, Los Angeles, California, 90024.

50 reprints are provided free for each article; additional copies may be obtained at cost in multiples of 50 .

The Pacific Journal of Mathematics is published monthly. Effective with Volume 16 the price per volume (3 numbers) is $\$ 8.00$; single issues, $\$ 3.00$. Special price for current issues to individual faculty members of supporting institutions and to individual members of the American Mathematical Society: $\$ 4.00$ per volume; single issues $\$ 1.50$. Back numbers are available.

Subscriptions, orders for back numbers, and changes of address should be sent to Pacific Journal of Mathematics, 103 Highland Boulevard, Berkeley, California, 94708.

PUBLISHED BY PACIFIC JOURNAL OF MATHEMATICS, A NON-PROFIT CORPORATION

Printed at Kokusai Bunken Insatsusha (International Academic Printing Co., Ltd.), 7-17, Fujimi 2-chome, Chiyoda-ku, Tokyo, Japan. 


\section{Pacific Journal of Mathematics}

\section{Vol. 37, No. $3 \quad$ March, 1971}

Mohammad Shafqat Ali and Marvin David Marcus, On the degree of the

minimal polynomial of a commutator operator ................ 561

Howard Anton and William J. Pervin, Integration on topological

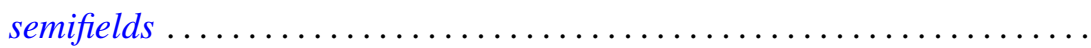

Martin Bartelt, Multipliers and operator algebras on bounded analytic

functions .................................... 575

Donald Earl Bennett, Aposyndetic properties of unicoherent continua ...... 585

James W. Bond, Lie algebras of genus one and genus two ............. 591

Mario Borelli, The cohomology of divisorial varieties ............... 617

Carlos R. Borges, How to recognize homeomorphisms and isometries ....... 625

J. C. Breckenridge, Burkill-Cesari integrals of quasi additive interval

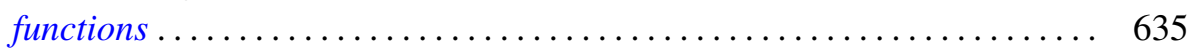

J. Csima, A class of counterexamples on permanents ................ 655

Carl Hanson Fitzgerald, Conformal mappings onto $\omega$-swirly domains . . . . . . 657

Newcomb Greenleaf, Analytic sheaves on Klein surfaces .............. 671

G. Goss and Giovanni Viglino, C-compact and functionally compact

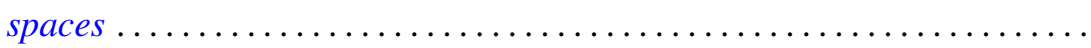

Charles Lemuel Hagopian, Arcwise connectivity of semi-aposyndetic plane

continua ..................................... 683

John Harris and Olga Higgins, Prime generators with parabolic limits ...

David Michael Henry, Stratifiable spaces, semi-stratifiable spaces, and their

relation through mappings .......................

Raymond D. Holmes, On contractive semigroups of mappings ........... 701

Joseph Edmund Kist and P. H. Maserick, BV-functions on semilattices ....... 711

Shûichirô Maeda, On point-free parallelism and Wilcox lattices ........... 725

Gary L. Musser, Linear semiprime $(p ; q)$ radicals ................. 749

William Charles Nemitz and Thomas Paul Whaley, Varieties of implicative

semilattices..................................... 759

Jaroslav Nešetřil, A congruence theorem for asymmetric trees ............ 771

Robert Anthony Nowlan, A study of $H$-spaces via left translations .......... 779

Gert Kjærgaard Pedersen, Atomic and diffuse functionals on a $C^{*}$-algebra ... 795

Tilak Raj Prabhakar, On the other set of the biorthogonal polynomials

suggested by the Laguerre polynomials...

801

Leland Edward Rogers, Mutually aposyndetic products of chainable

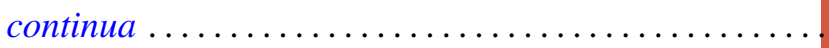

Frederick Stern, An estimate for Wiener integrals connected with squared

error in a Fourier series approximation.

Leonard Paul Sternbach, On k-shrinking and k-boundedly complete basic

sequences and quasi-reflexive spaces .................... 817

Pak-Ken Wong, Modular annihilator $A^{*}$-algebras ........ 